\title{
Competence, specification and induction of Pax-3 in the trigeminal placode
}

\author{
Clare V. H. Baker*, Michael R. Stark ${ }^{\star} \neq$, Christophe Marcelle $\$$ and Marianne Bronner-Fraserף \\ Division of Biology, Beckman Institute 139-74, California Institute of Technology, Pasadena, California 91125, USA \\ *The first two authors contributed equally to this work and are listed in alphabetical order \\ FPresent address: Department of Neurobiology and Anatomy, University of Utah School of Medicine, Salt Lake City, Utah 84132 USA \\ §Present address: Developmental Biology Institute of Marseille, LGPD-UMR CNRS 6545, Campus de Luminy, Case 907, 13288 Marseille Cedex 9 , France \\ IAuthor for correspondence (e-mail: mbronner@caltech.edu)
}

Accepted 19 October; published on WWW 3 December 1998

\section{SUMMARY}

Placodes are discrete regions of thickened ectoderm that contribute extensively to the peripheral nervous system in the vertebrate head. The paired-domain transcription factor Pax-3 is an early molecular marker for the avian ophthalmic trigeminal (opV) placode, which forms sensory neurons in the ophthalmic lobe of the trigeminal ganglion. Here, we use collagen gel cultures and heterotopic quailchick grafts to examine the competence, specification and induction of Pax-3 in the opV placode. At the 3-somite stage, the whole head ectoderm rostral to the first somite is competent to express Pax-3 when grafted to the opV placode region, though competence is rapidly lost thereafter in otic-level ectoderm. Pax-3 specification in presumptive opV placode ectoderm occurs by the 8 -somite stage, concomitant with robust Pax-3 expression. From the 8-somite stage onwards, significant numbers of cells are committed to express Pax-3. The entire length of the neural tube has the ability to induce Pax-3 expression in competent head ectoderm and the inductive interaction is direct. We propose a detailed model for Pax-3 induction in the opV placode.

Key words: Pax-3, Induction, Trigeminal, Placode, Cranial ganglia, Chick

\section{INTRODUCTION}

In vertebrate embryos, the formation of the sense organs (nose, eyes, ears and lateral line) and the cranial sensory ganglia is substantially dependent on the contribution of cells from cranial ectodermal placodes (reviewed by Webb and Noden, 1993; Le Douarin et al., 1986). Placodes, discovered over a century ago by van Wijhe (1883), are focal regions of thickened columnar epithelium that form in characteristic positions in the cranial ectoderm. The adenohypophyseal placode forms the anterior pituitary gland, while the lens placode forms the crystallin-secreting lens cells of the eye. The olfactory, otic and lateral line placodes give rise to the nose, ear and lateral line system, respectively. These three placodes form a wide variety of cell types, including ciliated sensory receptors, sensory neurons, neuroendocrine cells, glia and other supporting cells (see Webb and Noden, 1993; Muske, 1993 for reviews). The trigeminal and epibranchial (geniculate, petrosal and nodose) placodes give rise only to sensory neurons in cranial sensory ganglia (D’Amico-Martel and Noden, 1983).

The mechanisms underlying the formation of different placodes remain unclear, because there have been few early markers for placodes until very recently. Information on placode induction is essentially only available for the lens, olfactory and otic placodes, which form morphologically identifiable derivatives. Classical embryological grafting experiments in amphibian embryos have shown that the induction of the lens, nose and ear is a lengthy, cumulative process that begins during gastrulation (reviewed by Jacobson and Sater, 1988; Grainger, 1992, 1996). Induction of all three organs involves a series of different inducers from different tissues, including underlying mesoderm or endoderm and adjacent neural tissue (ibid.). Owing to this complexity, and in the absence of early molecular markers, it is very difficult to identify candidate inducing molecules through their expression patterns or to design assays to identify inducing molecules directly (as stressed by Gurdon, 1987). Today, however, such early markers have been identified for many placodes. Pax-6 is expressed in the lens and olfactory placodes (Walther and Gruss, 1991), Nkx5-1 and Pax-2 in the otic placode (Herbrand et al., 1998), c-Kit-related kinase $1, M s x-2$ and $D l x-3$ in lateral line placodes (Baker et al., 1995; Metscher et al., 1997), Pax3, FREK and neurogenin-1 in the trigeminal placode (Stark et al., 1997; Sommer et al., 1996) and neurogenin-2 in the epibranchial placodes (Sommer et al., 1996). Very recently, Otx-2, Sox-3 and Pax-6 have been identified as markers for presumptive lens ectoderm in some of the earliest stages of lens placode induction (Zygar et al., 1998; also see Altmann et al., 1997). The use of these early markers, in combination with classically defined information on the location and timing of induction, raises the exciting prospect of being able to identify the molecules involved in placode induction.

The availability of molecular markers also means that it is possible to study the induction of placodes lacking 
morphologically identifiable derivatives, i.e. the trigeminal and epibranchial placodes. In a previous study (Stark et al., 1997), we identified the paired-domain homeobox transcription factor Pax-3 as an early marker of the avian ophthalmic trigeminal (opV) placode. This placode gives rise to sensory neurons in the ophthalmic lobe of the trigeminal ganglion (opV). We showed using ablation and barrier implantation experiments that a diffusible signal from the midbrain/rostral hindbrain is necessary for Pax-3 induction or maintenance in the opV placode (Stark et al., 1997). In order to understand the cellular and molecular mechanisms underlying the induction of the opV placode, it is necessary to analyse the spatial and temporal organisation of Pax-3 induction in presumptive opV placode ectoderm. Here, we have used collagen gel cultures and classical embryological grafting techniques to determine: (1) which regions of the early embryo are competent to express Pax-3 protein in response to the Pax-3-inducing activity, (2) when presumptive opV placode ectoderm is specified and committed to express Pax-3 protein; and (3) where and when the Pax-3-inducing activity is present. We present a detailed model for Pax-3 induction in the opV placode.

\section{MATERIALS AND METHODS}

\section{Quail-chick grafts}

Fertilized quail (Coturnix coturnix japonica) and chick (Gallus gallus domesticus, White Leghorn) eggs were obtained from commercial sources and incubated at $38^{\circ} \mathrm{C}$ in a humidified atmosphere. Embryos were staged according to the number of somites formed. A window was cut in the quail shell and a 1:10 mixture of India ink and Ringer's solution injected into the sub-blastodermal cavity to reveal the embryo. The vitelline membrane in the region of the surgery, and the ectodermal region to be grafted, were removed using a pulled glass needle. The chick embryo host was similarly prepared and the ectoderm from the region of the graft site was removed immediately before transfer of the donor ectoderm.

\section{Collagen gel cultures}

Ectoderm explants for collagen gel cultures were removed as described above, or after enzymatic treatment to enable clean isolation of the ectoderm from underlying mesenchyme or neural crest cells. Other embryonic tissues (neural tube, mesoderm) were also dissected out following enzymatic treatment. Briefly, explanted quail and chick embryos were incubated in $1.5 \mathrm{mg} / \mathrm{ml}$ Dispase (Boehringer Mannheim) in DMEM with $20 \mathrm{mM}$ Hepes, $\mathrm{pH} 8.0$ for 20 minutes on ice and 15 minutes at $37^{\circ} \mathrm{C}$. After recovery on ice in complete medium (10\% horse serum, 15\% chick embryonic extract in DMEM) for at least 10 minutes, the embryos were removed into Ringer's solution and the desired tissue dissected out using pulled glass needles or 30 G1/2 syringe needles. Explants were stored on ice in complete medium until ready to be placed in collagen gel culture, when they were rinsed for 5-10 minutes on ice in Ringer's solution. Collagen matrix gels were prepared as previously described (Artinger and Bronner-Fraser, 1993) except that commercially produced collagen was used (Collaborative Research). The bottom layer (10 $\mu$ l collagen) was allowed to set for 20 minutes at room temperature before the explants were added. The top layer ( $4 \mu$ collagen) was allowed to set for 15 minutes at room temperature before addition of F-12 medium plus N-2 supplements (Gibco-BRL). Cultures were incubated at $38^{\circ} \mathrm{C}$, $5 \% \mathrm{CO}_{2}$ for $9-24$ hours.

\section{Immunohistochemistry}

Embryos and collagen gels were collected after 0 to 27 hours of incubation, fixed in $4 \%$ paraformaldehyde for 2 hours at room temperature and processed for cryostat sectioning as described by Sechrist et al. (1995). 12-16 $\mu \mathrm{m}$ sections were mounted on Superfrost ${ }^{\circledR}$ Plus glass slides (Fisher) and the gelatin removed by treating the slides in phosphate-buffered saline (PBS) at $42^{\circ} \mathrm{C}$ for 5 minutes. Slides were incubated 2-4 hours in primary antibody solution, and 1-2 hours in secondary antibody solution, with three 5minute washes after each incubation. For collagen gel specimens, DAPI was present at $10 \mu \mathrm{g} / \mathrm{ml}$ in the final wash. Slides were mounted in Fluoromount-G (Southern Biotechnology Associates, Inc.) and viewed by fluorescent microscopy. Only those chimeras that had successfully incorporated the donor graft were scored for Pax-3expressing quail cells. All sections were scored blind.

Antibodies were diluted in antibody buffer consisting of $0.1 \%$ BSA and $0.1 \%$ Triton in PBS. Primary antibody solution consisted of $1: 1$ QCPN supernatant (Developmental Hybridoma Bank, University of Iowa) and 1:5000 Pax-3 ascites (C. M., T. Wong, M. R. S., C. Ordahl and M. B.-F., unpublished data) in antibody buffer. Secondary antibody solution for quail-chick chimeras and collagen gel cocultures consisted of 1:400 goat anti-mouse IgG1-TRITC and 1:200 goat anti-mouse IgG2a-FITC (Southern Biotechnology Associates, Inc.) in antibody buffer. Secondary antibody solution for collagen gel specimens (quail tissue only) consisted of 1:300 goat anti-mouse IgGHi-FITC (Antibodies Inc.) in antibody buffer. For neurofilament staining, primary antibody solution consisted of 1:300 monoclonal antibody RMO 270.3 (Lee et al., 1987; gift of Dr Virginia Lee); secondary antibody solution consisted of 1:300 goat anti-mouse IgGHi-FITC (Antibodies, Inc.).

\section{RESULTS}

\section{Time course of Pax-3 protein expression in opV placode ectoderm}

Pax-3 mRNA can be detected in the dorsal neural tube and in the cranial neural folds and immediately adjacent surface ectoderm as early as the 3-somite stage (3-ss) in quail and chick embryos. As development proceeds, high levels of Pax-3 mRNA can be detected only in opV placode cells (Stark et al., 1997). Here, we used an anti-Pax-3 antibody to study the time course of Pax-3 protein expression. Pax-3 protein, in contrast to Pax-3 mRNA, cannot be detected above background in the dorsal neural tube, the neural crest or in the surface ectoderm prior to the 6-ss in quail or chick embryos. In 7-ss quail embryos, low levels of Pax-3 protein can be detected in ectoderm immediately adjacent to the midbrain, and also in neural crest cells migrating immediately beneath this ectoderm, with a few cells expressing slightly higher levels (Fig. 1A). In 8- to 9-ss quail embryos, neural crest cells in the presumptive opV placode region express roughly the same level of Pax-3 protein, while a few additional surface ectoderm cells begin to express higher levels (data not shown). In 10-ss embryos, several surface ectoderm cells express high levels of Pax-3 protein, while the underlying neural crest cells express comparatively lower levels (Fig. 1B). In 12- to 15-ss embryos, high levels of Pax-3 protein are seen in opV placodal ectoderm. After the 15-ss, Pax-3 protein expression in neural crest cells becomes virtually undetectable (Fig. 1C,D). By the 19-ss, both surface ectoderm and several placode-derived cells in the mesenchyme (see Stark et al., 1997) express high levels of Pax3 protein (Fig. 1E). The first detectable Pax-3 protein expression in young chick embryos lags 1- to 2-ss behind expression in quail embryos, but otherwise chick and quail show identical Pax-3 expression patterns. 
It is important to note that paraxial mesoderm caudal to about the level of rhombomeres 2 and 3 (r2,3) also expresses Pax-3 mRNA (Stark et al., 1997) as well as low levels of Pax3 protein. This includes mesoderm around the otic vesicles at young stages (up to about the 10-ss) and presomitic and somitic mesoderm at all stages examined (data not shown; see Stark et al., 1997 for mRNA expression). Also, migrating neural crest cells rostral and caudal to the otic vesicle express Pax-3 mRNA and protein at an intermediate level during their migration. Therefore, grafts involving otic-level ectoderm and more caudal regions were only scored for Pax-3 expression in quail surface ectoderm i.e. in columnar epithelium at the surface of the embryo. At all stages examined, the antibody is specific for cells expressing Pax-3 mRNA; it does not crossreact with cells expressing other Pax family gene members, such as Pax-2expressing otic placode cells (C. M., T. Wong, M. R. S., C. Ordahl and M. B.-F., unpublished data).

\section{Only head ectoderm rostral to the first-formed somite is competent to express Pax-3}

In order to determine the spatial extent of competence to express Pax-3, surface ectoderm from various regions of 2- to 11 -ss quail donors was grafted into the presumptive opV placode next to the midbrain and rhombomere 1 (region A) of 3- to 8-ss chick hosts (Fig. 2A). This definition of the presumptive opV placode is based on the placodal fate-map of D'Amico-Martel and Noden (1983), our own previous quailchick grafting experiments (Baker et al., 1997) and the early Pax-3 mRNA expression pattern in the cranial ectoderm (Stark et al., 1997). None of the ectodermal regions grafted into the presumptive opV placode, except presumptive opV placode itself at later stages, normally express Pax-3 mRNA (Stark et al., 1997). Chimeras were scored for Pax-3-positive quail cells after 15-24 hours of incubation. The results are presented graphically in Fig. 2B.

\section{Pre-otic ectoderm}

Forebrain-level ectoderm from 2- to 4-ss donors gave rise to many (>20) Pax-3-positive cells in all cases (F-A grafts). Although olfactory placode ectoderm, which fate-maps to the neural folds at the 3-ss (Couly and Le Douarin, 1985), goes on to express Pax-3 mRNA, 2- to 4-ss forebrain-level ectoderm does not express Pax-3 mRNA (Stark et al., 1997).

Isotopic grafts of midbrain-level ectoderm from 4- to 7-ss donors gave rise to many (>20) Pax-3-positive cells in all cases (A-A grafts; Fig. 2C-E). Ectoderm lateral to rhombomeres 2 and 3 (r2,3) from 6- to 9-ss donors gave rise to some (5-20) Pax-3-positive cells in $86 \%$ of cases (B-A grafts; donors younger than 6-ss could not be used as the rhombomere boundaries could not be distinguished). Although r2,3-level ectoderm does not express Pax-3 mRNA in unoperated embryos (Stark et al., 1997), and this ectoderm does not contribute cells to the opV ganglion (D'Amico-Martel and Noden, 1983), we confirmed that it does not normally give rise to Pax-3-positive cells by performing isotopic grafts at this level: Pax-3-positive quail cells were never seen in the resultant chimeras $(n=5)$.

\section{Otic-level ectoderm}

Otic-level ectoderm was defined in 2- to 3-ss embryos as the ectoderm rostral to the first-formed somite, based on the fate- map of Couly and Le Douarin (1990) and on our own isotopic grafts (data not shown). Ectoderm from the otic region of 2- to 3 -ss donors gave rise to a few $(<10)$ Pax-3-positive quail ectoderm cells in $88 \%$ of cases (C-A grafts). In contrast, oticlevel ectoderm from older embryos (4- to 9-ss donors) never formed Pax-3-positive cells. This suggests that the competence of otic-level ectoderm to express Pax-3 is rapidly lost after the 3 -ss. Ectopic quail-derived otic vesicle-like structures were often found after otic-level ectoderm grafts from older embryos. In isotopic grafts of otic-level ectoderm from 3- to 4-ss donors, no Pax-3-positive quail cells were seen $(n=3)$, again confirming that this region does not normally give rise to Pax-3-expressing cells.

\section{Postotic ectoderm}

Presumptive nodose placode ectoderm was defined as the ectoderm overlying and lateral to the first two-formed somites (D'Amico-Martel and Noden, 1983). Ectoderm from the presumptive nodose placode region of 3- to 6-ss donors never gave rise to Pax-3-positive ectoderm cells (D-A grafts). Ectoderm from the trunk of 3- to 11-ss donors, whether from over the somites or lateral plate mesoderm, lateral to the open neural plate, or lateral to Hensen's node, likewise never gave rise to Pax-3-positive ectoderm cells (L/M-A grafts).

In summary, only head ectoderm rostral to the first-formed somite is competent to express Pax-3 when grafted adjacent to the midbrain. Competence is highest in more rostral ectoderm (forebrain- and midbrain-level ectoderm). Competence at the otic level is lost after the 3 -ss, while competence at the r2,3 level is retained until at least the 9-ss (the latest stage tested).

\section{Competence to express Pax-3 is present at gastrula stages}

In order to determine how early in development we can detect competence to express Pax-3, we took prospective ectoderm (García-Martinez et al., 1993) from the lateral margins of Hamburger-Hamilton (HH) stage $3+$ quail epiblasts (Hamburger and Hamilton, 1951) and grafted it into the presumptive opV placode region of 6- to 8-ss chick hosts. In all cases, the grafted tissue formed at least some (3-30) Pax-3positive cells $(n=5)$; however, these were always localised to particular regions of the graft, usually its caudal margin. Grafts taken from the lateral margins of $\mathrm{HH}$ stage 5 embryos formed many (>20) Pax-3-positive cells in $75 \%$ of 3 - to 5-ss hosts $(n=4)$. Hence, competence to express Pax-3 is present, at least to some extent, in the epiblast as early as $\mathrm{HH}$ stage $3+$.

\section{Pax-3 specification does not occur significantly before Pax-3 expression}

A tissue is specified to follow a particular developmental pathway if it follows that pathway when isolated from the embryo and cultured in a neutral medium, i.e. in the absence of other embryonic signals (Slack, 1991). In order to test when presumptive opV placode ectoderm becomes specified to express Pax-3, we explanted midbrain-level ectoderm pieces from 3- to 13-ss quail donors and cultured them in collagen gels without serum for 9-22 hours. The explanted region was taken a little lateral to the midbrain to avoid accidental extirpation of neural tissue, and is devoid of Pax-3 mRNA expression at the 3-ss (Stark et al., 1997). Couly and Le Douarin (1990) found the anlage of the opV placode to be 

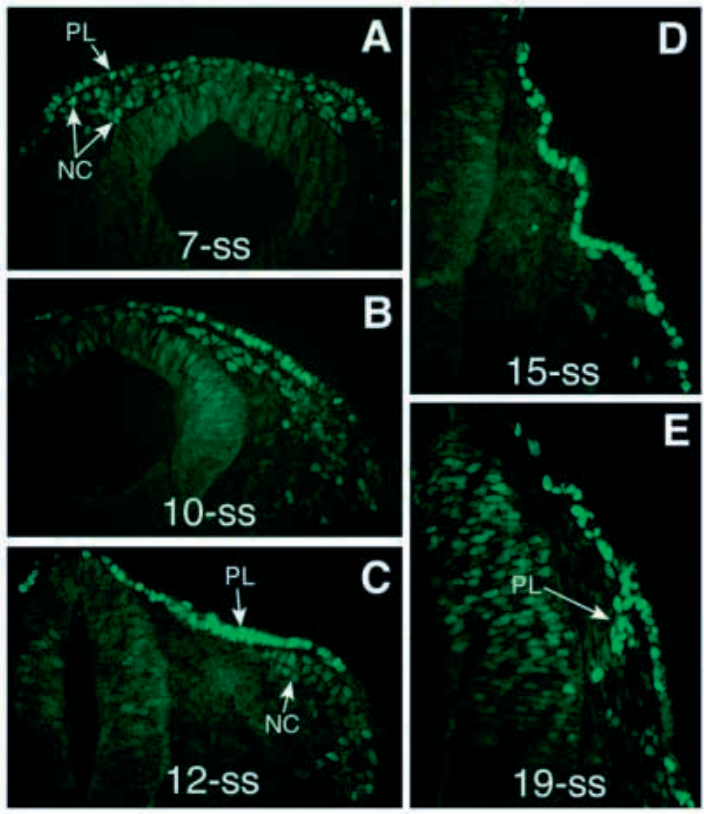

contained entirely within the neural folds at the 3 -somite stage (3-ss). In our hands, however, competent ectoderm grafted next to the midbrain at the 3 -ss forms neurons in the opV ganglion (data not shown). The results were pooled into four timeperiods: 3- to 4-ss, 5- to 7-ss, 8- to 9-ss and 10- to 13-ss. Since one somite-stage is approximately 1.5 hours, these periods are short relative to cell cycle time in the ectoderm, which is around 10 hours at this stage (Smith and Schoenwolf, 1987).
Fig. 1. Transverse sections through the midbrain region of quail (A-C) and chick (D,E) embryos stained with the anti-Pax-3 antibody to show the normal time course of Pax-3 protein expression in the opV placode. (A) 7-ss quail embryo, showing low levels of Pax-3 protein in the migrating neural crest (NC) and overlying presumptive placodal ectoderm (PL). (B) 10-ss quail embryo, showing low Pax-3 expression in migrating neural crest cells and higher expression in presumptive opV placode cells. (C) 12-ss quail embryo, showing high Pax-3 expression in the presumptive opV placode and low expression in some neural crest cells. (D) 15-ss chick embryo, showing high Pax3 expression in the opV placode. (E) 19-ss chick embryo, showing high Pax-3 expression in the opV placode and in migrating placodal cells (see Stark et al., 1997). NC, neural crest cells; PL, placode cells.

After sectioning the collagen gels, we immunostained them with the anti-Pax-3 antibody to identify Pax-3-expressing cells, and labelled them with DAPI to follow cell death. We found that after 12 hours of incubation, there was substantial cell death in any adhering mesenchyme or neural crest, but the ectoderm remained healthy. The results are presented graphically in Fig. 3A. A few $(<5)$ faintly Pax-3-positive cells were seen in $14 \%$ of 3 - to 4 -ss explants, and a few (1-6) cells were seen in $24 \%$ of 5 - to 7 -ss explants. At the 8 - to 9 -ss, $55 \%$ of explants contained at least a few (1-10) Pax-3-positive cells, most of which showed intermediate to bright staining (Fig. 3B). Half of the 8- to 9-ss embryos were treated with dispase to allow ectoderm removal without adhering neural crest cells, which migrate beneath the explanted midbrain-level ectoderm from the 8-ss onwards (see Fig. 1). The presence or absence of neural crest cells had no effect on the results $(58 \%$ of untreated explants yielded Pax-3-positive cells, compared with 52\% of explants from dispase-treated embryos). Given the in vivo
Fig. 2. Analysis of the distribution of ectoderm competent to express Pax-3 in response to the midbrain-level-inducing activity. (A) Different ectoderm regions from quail donors were grafted at the midbrain level of chick hosts. (B) Histogram of the results. Each column represents the percentage of chimeras with Pax-3-positive quail cells; the total number of grafts performed $(n)$ is marked at the top of each column. Shown are forebrain-level grafts (F-A, 100\%), isotopic midbrain-level grafts (A-A, $100 \%$ ), r2-3-level ectoderm grafts (B-A, $86 \%$ ), oticlevel ectoderm grafts (C-A, 2- to 3-ss donors, $88 \%$ : C-A, 4-ss and older donors; $0 \%$ ), and trunk-level ectoderm grafts (L/M-A, 0\%). Forebrain- and midbrain-level grafts (F-A and A-A) yielded many more Pax-3-positive cells per graft than did r2,3- or 2- to 3-ss otic-level grafts (B-A and C-A).

(C-E) Transverse section through the midbrain region of a chick host collected 20 hours after receiving an isotopic graft of 5-ss midbrain-level quail ectoderm. (C) FITC-labelled Pax-3-positive cells appear green. (D) TRITC-labelled QCPN-positive quail cells appear red. (E) Pax-3-positive quail cells appear yellow when the green and red channels are merged. Pax-3-positive chick cells can be seen in the ectoderm dorsal to the Pax-3-positive quail ectoderm.
A
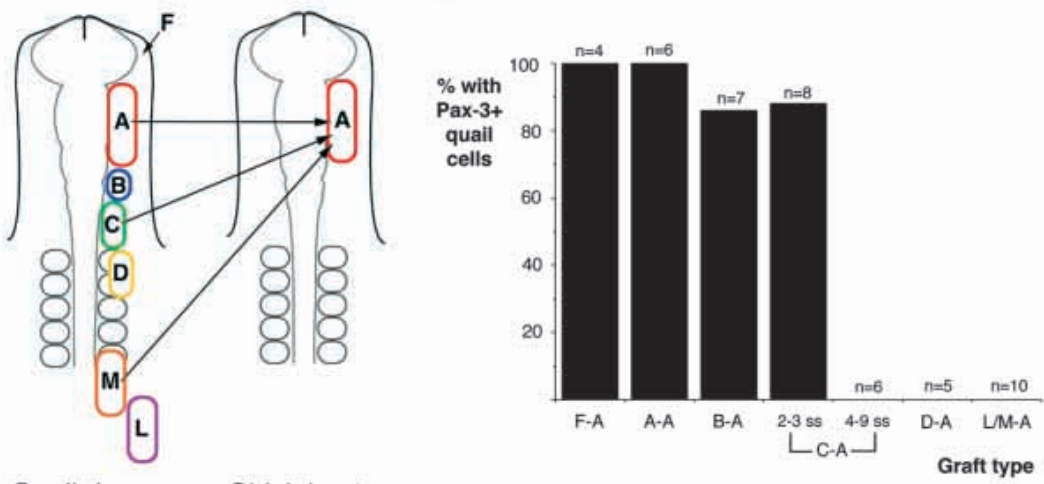

Quail donor

Chick host
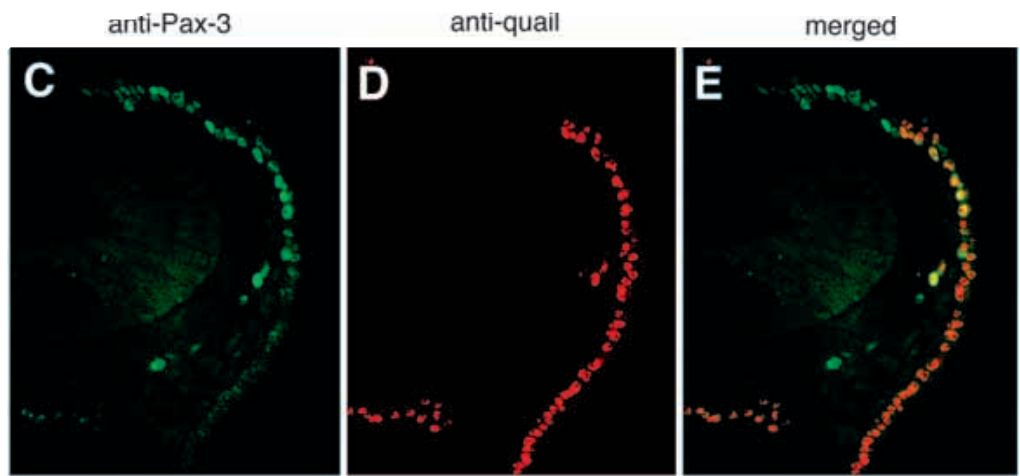
A
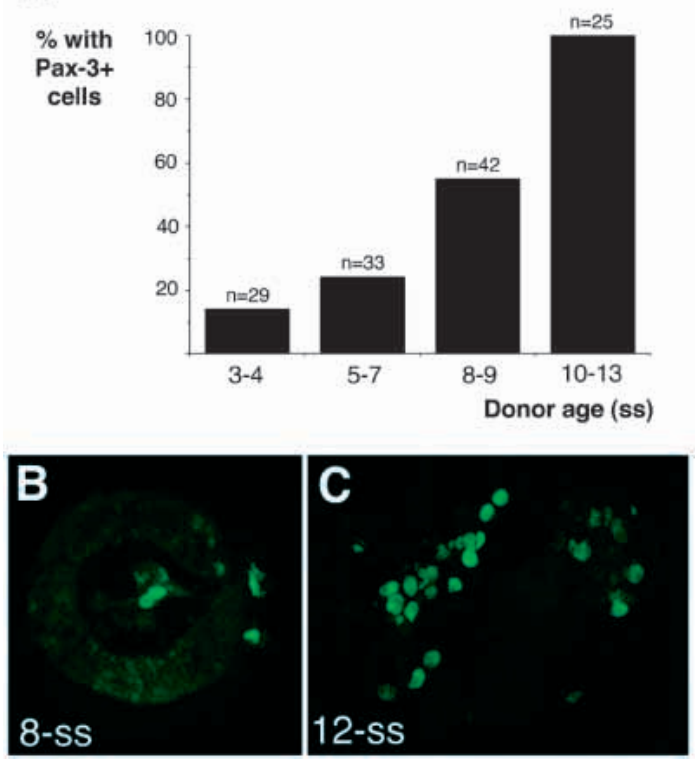

Fig. 3. Time course of specification of presumptive opV placode ectoderm to express Pax-3 protein. Midbrain-level ectoderm explants were cultured in collagen gels without serum for 9-22 hours, sectioned and scored for Pax-3-positive cells. (A) Histogram showing the percentage of collagen gel-cultured explants with Pax-3positive cells at different donor stages. The number of explants $(n)$ is listed at the top of each column. Before the 8- to 9-ss, only a few Pax-3-positive cells are seen per explant. From the 8- to 9-ss, increasing numbers of cells with higher levels of expression are seen. (B) Pax-3 protein expression in dispase-treated 8-ss midbrain-level ectoderm cultured for 20 hours. Dispase treatment to remove underlying neural crest cells had no effect on Pax-3 expression (see text). (C) Pax-3 protein expression in isolated 12-ss midbrain-level ectoderm cultured for 18 hours.

expression pattern (Fig. 1), the 8- to 9-ss is probably when Pax3 protein begins to be expressed at higher levels in the explanted region. By the 10- to 13-ss, all explants contained at least a few, and usually many (2-20 or more) Pax-3positive cells, most of which stained brightly (Fig. 3C). The intensity of staining and the number of Pax3-positive cells increased with the age of the explant: brightly staining cells, and explants containing more than ten Pax-3-positive cells, were only found after the 8 -ss. Thus, specification of significant numbers of cells in the explanted region occurs from the 8-ss onwards, which is when observable Pax-3 protein expression begins in this region in vivo.

\section{Presumptive opV placode ectoderm is committed to express Pax-3 from the 8-ss}

A tissue is committed to follow a particular developmental pathway if it still follows that pathway when grafted elsewhere in the embryo, i.e. when it is exposed to other embryonic signals. In order to test when presumptive opV placode ectoderm becomes committed to express Pax-3, we grafted midbrainlevel ectoderm from 4- to 11-ss quail embryos into the trunk of chick hosts, at a distance from the neural tube, where we expected no inducing activity to be present. The grafts were placed over the intermediate and lateral plate mesoderm, at the level of the first newly formed somite or the rostral segmental plate (Fig. 4A). The chimeras were allowed to develop for 9-27 hours and then scored for Pax-3-positive quail cells. The results are presented graphically in Fig. 4B. Already at the 4-ss, 25\% of grafts yielded a few (<10) Pax-3positive cells. As the donor age increased, the percentage of grafts yielding Pax-3-positive cells also increased, until by the 10- to 11-ss, all grafts yielded Pax-3-positive cells. This apparently linear progression, however, hides a significant change in the number of Pax-3-positive cells per graft that occurs at the 8-ss. Before the 8 -ss, $96 \%$ of grafts yielded fewer than ten Pax-3-positive cells (the single exception was from a 7 -ss donor); after the 8 -ss, $88 \%$ of grafts yielded more than twenty Pax-3-positive cells. Hence commitment of significant numbers of cells in the explanted region occurs from the 8-ss onwards. We allowed several embryos that received grafts of 5- to 6-ss midbrain-level ectoderm to develop for 36 hours and immunostained sections with QCPN and an anti-neurofilament antibody. A few neurofilament-positive quail cells were seen in these embryos (data not shown), suggesting that the Pax-3positive cells also go on to express neuronal markers.

Any definitive analysis of commitment is difficult to achieve, because other Pax-3-inducing signals may be present at the graft site. To eliminate the possibility that Pax-3inducing signals are present in underlying lateral plate or adjacent paraxial mesoderm, we co-cultured 4- to 7-ss midbrain-level quail ectoderm pieces with isolated 10- to 13ss chick lateral plate mesoderm or somitic mesoderm, in collagen gels without serum for 11-14 hours. When co-cultured with lateral plate mesoderm, $8 \%$ of explants yielded a few $(<5)$ Pax-3-positive quail cells $(n=13)$. When co-cultured with somitic mesoderm (segmental plate and somites), $29 \%$ of explants yielded a few (<5) Pax-3-positive quail cells $(n=14)$. Given that 5- to 7-ss midbrain-level ectoderm cultured alone yielded Pax-3-positive cells in $24 \%$ of cases (Fig. 3A), it seems unlikely that the underlying mesoderm is involved in inducing Pax-3 in lateral trunk grafts of unspecified midbrain-level ectoderm.
A

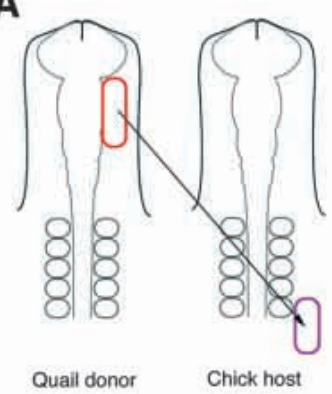

B

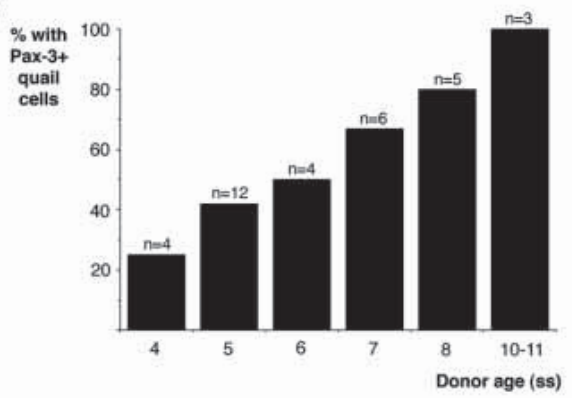

Fig. 4. Time course of commitment of presumptive opV placode ectoderm to express Pax-3 protein. Midbrain-level ectoderm was grafted from quail donors into the trunk of chick hosts, over the intermediate and lateral plate mesoderm. Chimeras were sectioned and scored for Pax-3-positive quail cells. (A) Histogram showing the percentage of chimeras with Pax-3-positive quail cells after grafts from different donor stages. The number of grafts $(n)$ is listed at the top of each column. Before the 8-ss, only a few Pax-3-positive cells are seen per embryo. From the 8-ss onwards, many Pax-3-positive cells are seen. 


\section{The Pax-3-inducing activity is present at all axial levels}

We previously showed that blocking normal interactions between the neural tube and the presumptive opV placode in 4- to 8-ss chick embryos prevents placodal Pax-3 mRNA expression, implicating the neural tube in opV placode induction (Stark et al., 1997). In order to map the spatial extent of the inducing activity, we grafted midbrain-level ectoderm (region A) from 3- to 7-ss donor quail embryos next to the neural tube of host chick embryos at different axial levels (Fig. 5A). At the 3- to 7-ss, midbrain-level ectoderm is essentially unspecified: combining the specification results described above for 3- to 4-ss and 5- to 7-ss explants shows that only $19 \%$ of 3- to 7-ss explants contained any (1-6) Pax-3-positive cells (total $n=62$ ). Thus this ectoderm can be used to assay the distribution of Pax-3-inducing activity. Chimeric embryos were collected after 13-27 hours of incubation and scored for Pax-3-positive quail cells. In grafts next to the hindbrain (3- to 7-ss hosts), quail tissue was scored independently for Pax-3 expression at each rhombomeric level, since the graft tissue often overlapped several rhombomeres. In trunk grafts, the donor ectoderm was usually placed over the first few newly formed somites or the segmental plate. Since somite formation is similar along the length of the axis, the ectoderm was thus grafted to sites that were considered to be at the same relative developmental stage. The results are depicted graphically in Fig. 5B.

Pax-3-positive quail cells (5-20 or more) were always found after grafts of midbrain-level ectoderm to the otic region (A-C grafts; Fig. 5C-E), nodose placode (A-D grafts) and trunk (A$\mathrm{M}$ grafts; Fig. 5F-H). These results suggest that the inducing activity is present not only in the midbrain, where the opV placode normally forms, but also in the hindbrain and in trunklevel neural tube. In all three types of grafts, fewer Pax-3positive quail cells were present than were seen in isotopic midbrain-level grafts (data not shown). This suggests either that less inducing activity is present in the hindbrain and trunk, or that the grafted quail cells respond to other signals and become committed to a different fate. For example, when grafts were positioned precisely at the level of the otic placode, Pax3-negative quail cells were often incorporated into the host otic vesicle (Fig. 5E). The long-term fate of heterotopically grafted opV placode cells will be addressed in detail in a subsequent paper.

When 4- to 6-ss midbrain-level ectoderm was grafted next to r2,3 (A-B grafts), only $78 \%$ of grafts contained Pax-3positive cells. Again, there were fewer Pax-3-positive quail cells than in isotopic midbrain-level grafts (data not shown). Even 8-ss midbrain-level ectoderm, which is already beginning to express Pax-3 in the embryo, sometimes did not express Pax3 when grafted to the r2,3 level (two out of four grafts were Pax-3-negative). R2,3-level ectoderm is competent to express Pax-3 when grafted into the midbrain or into the trunk (see later), but it does not express Pax-3 in normal development. This may suggest either that the level of inducer is reduced at the r 2,3 level, or that an inhibitor is present in this region.

\section{At midbrain levels, the inducing activity decreases after the 8-ss}

Young (2- to 3-ss) otic-level ectoderm is competent to express Pax-3 in response to the midbrain-level-inducing activity of 3- to 6-ss hosts, as described above. However, when 2- to 3-ss otic-level ectoderm was grafted into 9 - to 12 -ss hosts, only $14 \%$ formed Pax-3-positive cells $(n=7$; the single positive case was grafted into a 9-ss host). This may suggest that the level of the inducing activity in the midbrain decreases after the 8-ss.

\section{The neural tube induces Pax-3 directly in competent ectoderm}

We previously showed that the midbrain/rostral hindbrain is necessary for Pax-3 mRNA induction in the opV placode, as a barrier placed between the neural tube and ectoderm in this region eliminated placodal Pax-3 expression (Stark et al., 1997). We could not tell from these experiments whether the neural tube-derived-inducing activity acts directly on the ectoderm or indirectly via other tissues. We now tested whether the neural tube is sufficient to induce Pax-3 protein directly in presumptive opV placode ectoderm, by co-culturing unspecified midbrain-level quail ectoderm with isolated chick neural tube in collagen gels without serum for 11-18 hours.

In co-cultures with 4- to 8-ss midbrain, 3- to 7-ss midbrainlevel quail ectoderm formed 10-20 Pax-3-positive cells in 46\% of cases ( $n=13$; Fig. 6A-C). As described above, 3- to 7-ss ectoderm cultured alone forms a few (1-6) Pax-3-positive cells in $19 \%$ of cases. Thus, many more Pax-3-positive quail cells were seen after co-culture with midbrain than after culturing in isolation. We conclude that the midbrain can directly induce Pax-3 expression in presumptive opV placode ectoderm.

In co-cultures with 10- to 13-ss trunk neural tube, 3- to 7-ss midbrain-level ectoderm gave rise to ten to twenty or more Pax-3-positive cells in 55\% of cases ( $n=22$; Fig. 6D-F). Again, many more Pax-3-positive cells were seen per co-culture than when the ectoderm is cultured alone. The dorsoventral orientation of the trunk neural tube is evident in section, as the dorsal neural tube faintly expresses Pax-3 (see Fig. 6D). When the results are broken down into dorsally versus ventrally located ectoderm pieces, it is clear that the Pax-3-inducing activity is localised to the dorsal neural tube. $73 \%$ of dorsally located explants yielded Pax-3-positive cells $(n=11)$, compared with $18 \%$ of ventrally located explants $(n=11)$. Furthermore, Pax-3-positive cells seen in ventrally located explants were all in regions of the explant that extended dorsally and were adjacent to Pax-3-expressing regions of the neural tube; they were never seen next to the Pax-3-negative ventral neural tube. These results therefore demonstrate both that trunk neural tube can directly induce Pax-3 in competent ectoderm and that the inducing activity is localised to the dorsal neural tube.

\section{A few cells in r3- and young otic-level ectoderm are also specified to express Pax-3}

Since all head ectoderm rostral to the first somite is competent at the 3-ss to respond to the Pax-3-inducing activity, which is present throughout the neural tube (see above), we wondered whether any non-opV head ectoderm is ever specified to express Pax-3. We explanted r3- and otic-level ectoderm pieces from quail donors into collagen gels and cultured them without serum for 18-21 hours. R3-level ectoderm from 6- to 12-ss donors formed a few $(<5)$ Pax-3-positive cells in 36\% of cases $(n=28)$. Otic-level ectoderm from 2- to 3-ss donors formed a few $(<5)$ Pax-3-positive cells in $27 \%$ of cases $(n=11)$. These results show (1) that hindbrain-level ectoderm normally receives the inductive signal, as suggested by our analysis of the spatial 
extent of the inducing activity, and (2) that a very few cells in these regions are specified to express Pax-3. Older otic-level ectoderm (6- to 13-ss donors), which was previously shown to lack competence to express Pax-3 (see above), never formed Pax-3-positive cells in culture $(n=14)$. Therefore, this region of ectoderm has presumably received other signals by the 6-ss that forestall any Pax-3 expression. Similarly, since r3-level ectoderm does not express Pax-3 in vivo (as seen both by wholemount in situ hybridisation and after isotopic grafts; Stark et al., 1997; current results), Pax-3 expression at this level is prevented in vivo. As expected, trunk ectoderm overlying the lateral plate mesoderm (7- to 9-ss donors), which was previously shown to lack competence to express Pax-3 (see above), never gave rise to Pax-3-positive cells in culture $(n=10)$.

\section{DISCUSSION}

Our aim is to understand the cellular and molecular mechanisms underlying the formation of the trigeminal placode. We previously showed that the transcription factor Pax 3 is an early marker for the avian ophthalmic trigeminal (opV) placode and we identified a diffusible inducing activity from the midbrain/rostral hindbrain as being necessary for Pax3 induction in the opV placode (Stark et al., 1997). Here, we determined which regions of ectoderm in the embryo are competent to respond to Pax-3-inducing signals and when they are competent to do so. We also examined in detail the time course of specification and commitment of presumptive opV placode cells to a Pax-3-expressing fate. This enabled us to determine when and where the Pax-3-inducing activity appears and to demonstrate that the neural tube induces Pax-3 directly in competent ectoderm. This allows us to propose a detailed model for Pax-3 induction in the opV placode.

\section{Spatial and temporal distribution of competent ectoderm}

At the 3-ss, the entire head ectoderm rostral to the first somite is competent to express Pax-3 in response to the midbrainlevel-inducing activity, as shown by grafting different regions of ectoderm next to the midbrain. This is supported by our previous experiments showing normal Pax-3 mRNA expression after radical surface ectoderm ablation in the opV placode region (Stark et al., 1997), suggesting that adjacent ectoderm can respond to the Pax-3-inducing activity. Likewise, Hamburger (1961) showed that the opV ganglion forms after ablation of the opV placode.

Competence is lower in more caudal regions, however, and after the 3 -ss, it is lost altogether from the otic region. It is retained by some tissue that does not normally express Pax-3, such as r2,3-level ectoderm. Competence is present in prospective ectoderm early, during gastrulation. It is worth noting that competence is in all probability also induced or regulated by signalling molecules (see Streit et al., 1997). Its loss, for example in the otic region, may be due to commitment to a different fate.

Trunk ectoderm is not competent to respond to the midbrainlevel Pax-3-inducing activity. However, Vogel and Davies (1993) showed that trunk ectoderm is competent to form nodose-type neurons after grafting into the presumptive nodose placode region, at the level of the first two somites.
Importantly, this shows that competence to form one placode does not necessarily indicate competence to form all placodes.

\section{Specification to express Pax-3}

We explanted midbrain-level ectoderm at different times and tested when it was specified to express Pax-3 by culturing it in collagen gels without serum. We found that Pax-3 specification does not occur significantly before Pax-3 expression. In some embryos, a few cells in the explanted region are specified to express Pax-3 protein before the 6- to 7-ss stage, which is when Pax-3 protein expression is first seen in the embryo (Fig. 1). However, this specification may be concurrent with Pax-3 mRNA expression, which begins before Pax-3 protein expression (see above and Stark et al., 1997). Increasing numbers of cells are specified from the 8-ss onwards, which is probably when Pax-3 protein begins to be expressed in vivo at higher levels.

\section{Commitment to express Pax-3}

In order to test commitment, we grafted midbrain-level ectoderm to the alien environment of the trunk, over the lateral plate mesoderm and therefore at a distance from the neural tube. We expected that no inducing activity would be present in this location. We found that significant numbers of cells in midbrain-level ectoderm are committed to express Pax-3 after the 8-ss, which is similar to the result obtained in the specification experiments. At each stage, however, the percentage of explants yielding Pax-3-positive cells was greater when they were grafted into the embryo (commitment experiments) than when they were cultured alone (specification experiments). This may not be surprising given that many other signals are present in the embryonic environment, some of which may be able to induce or support Pax-3 expression. We eliminated the underlying mesoderm as a potential source of additional Pax-3-inducing activity by co-culturing unspecified midbrain-level ectoderm with isolated lateral plate or somitic mesoderm. The percentage of explants yielding Pax-3-positive cells was not significantly increased after co-culture with either tissue. However, we cannot completely exclude the possibility that other Pax-3-inducing signals are present in this ectopic location. These results highlight the difficulty involved in testing commitment towards a particular fate.

\section{Spatial and temporal distribution of Pax-3-inducing activity}

We were able to use the essentially unspecified nature of 3- to 7-ss midbrain-level ectoderm to analyse the distribution of the inducing activity in the embryo, by grafting it next to the neural tube at different axial levels and looking for Pax-3 induction. The inducing activity is present at all axial levels, although the fact that midbrain-level ectoderm sometimes did not express Pax-3 when grafted to the r2,3 level suggests that there may be less inducer, or perhaps an inhibitor, at this level (see below). We showed that the neural tube can directly induce Pax-3 in unspecified ectoderm by co-culturing such ectoderm with isolated midbrain or trunk neural tube. These data also revealed that the inducing activity in the trunk is localised to the dorsal neural tube.

Our previous data suggested that the Pax-3-inducing activity must be present in the midbrain before the 3-ss, since Pax-3 mRNA can be seen in the ectoderm immediately adjacent to the midbrain at the 3-ss (Stark et al., 1997). The level of the 
Fig. 5. Analysis of the distribution of inducing activity. (A) Unspecified midbrain-level ectoderm from 3- to 7-ss quail donors was grafted next to the neural tube at different axial levels. (B) Histogram of the results. Each column represents the percentage of chimeras containing Pax-3-positive quail cells; the total number of grafts performed is marked at the top of each column. Shown are isotopic midbrain-level grafts (A-A; 100\%), r2-3-level grafts (A-B; 78\%), otic-level grafts (A-C; $100 \%$ ), and trunk-level grafts adjacent to the neural tube, over the paraxial mesoderm (A-M; $100 \%)$. (C-E) Transverse section at the otic level of a chick host collected 18 hours after receiving a graft of 6-ss midbrain-level quail ectoderm. (C) FITC-labelled Pax-3-positive cells (green) reside near the neural tube, medial to the rostral margin of the otic vesicle (ov). hb, hindbrain. (D) TRITC-labelled QCPN-positive quail cells (red) are seen in the same region. (E) Merging the green and red channels shows that all the Pax-3-positive cells are quail cells (yellow), and that some Pax-3-negative quail cells have integrated into the developing otic vesicle. $(\mathrm{F}-\mathrm{H})$ Transverse section through the trunk region of a chick host collected 19 hours after receiving a graft of 6-ss midbrain-level quail ectoderm over the segmental plate. (F) FITC-labelled Pax-3-positive cells (green) reside within the surface ectoderm and dermomyotome (dm). scl, sclerotome; nt, neural tube. (G) TRITC-labelled QCPNpositive quail cells (red) reside in the ectoderm and underlying mesenchyme. (H) Merging the green and red channels shows that brightly staining Pax-3-positive cells in the ectoderm and underlying mesenchyme are quail-derived (yellow), and that host dermomyotome cells contain slightly lower levels of Pax-3 protein
A

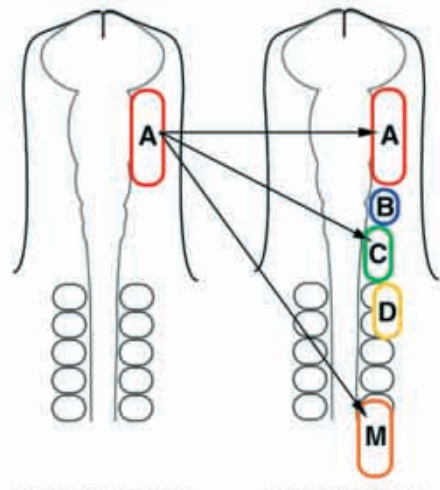

Quail donor
B

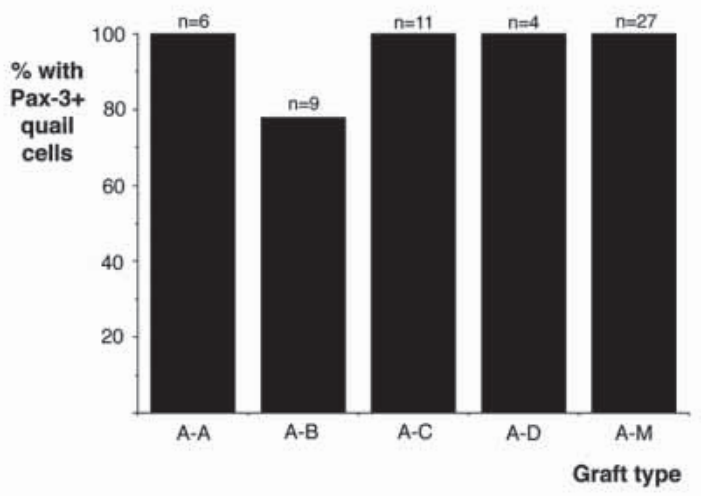

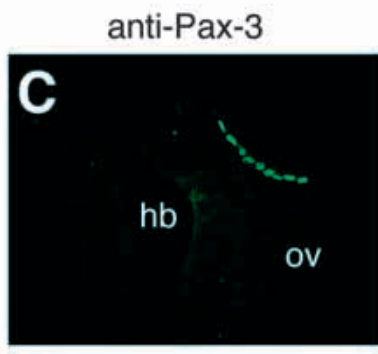
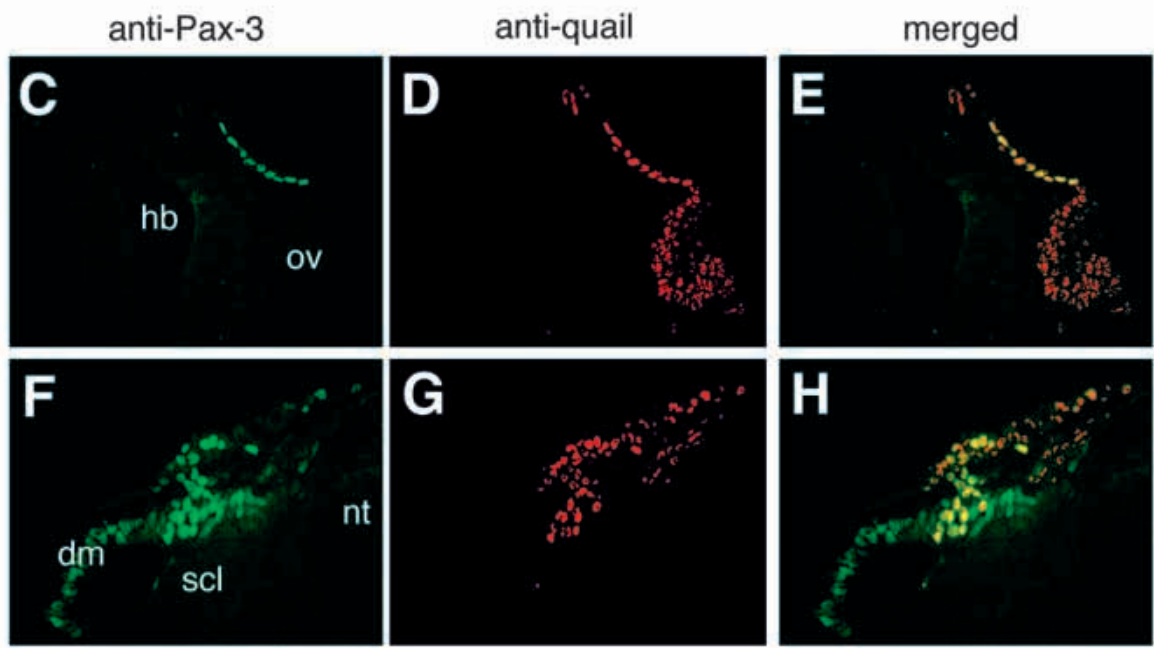

(A). dm, dermomyotome; hb, hindbrain; nt, neural tube; ov, otic vesicle; scl, sclerotome.

Fig. 6. The neural tube can directly induce Pax-3 expression in presumptive opV placode ectoderm. 3- to 7-ss midbrainlevel quail ectoderm was cocultured with isolated chick midbrain or trunk neural tube, in collagen gels without serum for 11-18 hours. (A-C) Section through a 14.5-hour co-culture of 5- to 6-ss midbrain-level quail ectoderm (i.e. from a pool of 5to 6-ss ectoderm pieces) with isolated 6-ss chick midbrain. (A) FITC-labelled Pax-3-positive cells appear green. (B) TRITClabelled QCPN-positive quail cells appear red. (C) When the green and red channels are merged, Pax-3-positive quail cells are seen as yellow (arrowhead). A few Pax-3-positive chick cells are also visible in the midbrain. (D-F) Section through a 13.5-hour co-culture of 4- to 7-ss midbrain-level quail ectoderm (i.e. from a pool of 4- to 7-ss ectoderm pieces) with isolated chick trunk neural tube. (D) FITC-labelled Pax-3-positive cells appear green. The dorsal neural tube (d) expresses Pax-3, while the ventral portion (v) does not. (E) TRITC-labelled QCPN-positive quail cells appear red. (F) When the green and red channels are merged, Pax-3-positive quail cells are seen as yellow (arrowhead). The dorsal neural tube is also faintly Pax-3-positive, with some scattered brightly staining cells. d, dorsal; v, ventral. 
inducing activity in the midbrain may decrease after the 8-ss, the stage at which more lateral midbrain-level ectoderm (the region explanted in our experiments) begins to express increasing amounts of Pax-3 protein in vivo. This is also the stage at which neural crest cells migrate beneath the ectoderm. However, we showed previously that deletion of the neural crest has no effect on Pax-3 expression in the opV placode (Stark et al., 1997). In addition, Pax-3 mRNA expression and at least some Pax-3 specification precede neural crest cell emigration (Stark et al., 1997; current results). Hence, the presence of the neural crest is not necessary for Pax-3 induction in the opV placode.

\section{How is Pax-3 induction restricted?}

In the present study, we have shown that a broad area of head ectoderm is competent to respond to the Pax-3-inducing activity at the 3-ss, that the inducing activity must be present in the midbrain before the 3 -ss and that the inducing activity is also broadly distributed along the entire neuraxis. How, then, is Pax-3 induction localised next to the midbrain and r1?

Firstly, the response is confined to competent ectoderm. Some regions lack competence from the earliest time tested: neither trunk ectoderm nor the nodose placode region (overlying and adjacent to the first two somites) is competent to express Pax-3. Other regions are competent at early stages, but subsequently lose competence. For example, otic-level ectoderm is competent at the 3-ss (and even specified to express Pax-3 to some extent), but loses competence after this stage. This loss of competence probably occurs as otic-level ectoderm becomes committed or more biased towards an otic fate.

Secondly, a localised inhibitor may prevent the response in certain regions of competent ectoderm. Ectoderm at the r2,3 level, which marks the caudal boundary of the opV placode, is competent to express Pax-3 until at least the 9-ss, by which time midbrain-r1-level ectoderm already expresses Pax-3 protein. A few cells in $\mathrm{r}$-level ectoderm are even specified to express Pax-3, but Pax-3 expression is never seen here in vivo. Either the level of inducer is much reduced at this level or an inhibitor of Pax-3 expression may be present. Another alternative, given that neural crest cells exiting at $\mathrm{r} 3$ undergo apoptosis in response to a signal from r4 (Graham et al., 1993, 1994, 1996), is that Pax-3-expressing cells in r3-level ectoderm also die in response to this signal. The presence of an inhibitor of some kind is also suggested by the fact that even midbrainlevel ectoderm sometimes does not express Pax-3 when grafted to the $\mathrm{r} 2,3$ level.

\section{Properties of embryonic induction}

Our results illustrate three general points about embryonic induction (Gurdon, 1987; Jacobson and Sater, 1988). Firstly, the state of the responding tissue is more important than the state of the inducing tissue in determining the outcome of the induction: the Pax-3-inducing activity is broadly distributed, but Pax-3 expression is nevertheless restricted to the midbrainr1 level. Secondly, both changes in competence and suppressive tissue interactions can limit the extent of the induction: caudal ectoderm regions lack competence altogether; otic-level ectoderm loses competence, while there may be an inhibitor at the r2,3-level. Thirdly, the same tissue can receive several different inductive signals simultaneously, and may be specified in some degree towards a number of different fates: some cells in $\mathrm{r} 3-$ and otic-level ectoderm are specified to express Pax-3. Induction is a cumulative process. The eventual fate adopted by a tissue therefore depends both on its history, i.e. signals received in the past that bias it towards a particular fate, and on signals received at any given time.

\section{Are placodes derived from a common anlage?}

It is possible that the ability of early placodal ectoderm to respond to many different placode-inducing signals reflects its derivation from a common anlage. In frog embryos, Knouff (1935) identified a 'primitive placodal thickening' between the neural crest and epidermis, from which all the placodes subsequently arise. In the Atlantic cod embryo, Miyake et al. (1997) found a continuous dorsal ectodermal thickening from the forebrain to the hindbrain that subsequently separates into three independent placodes. In mouse embryos, Verwoerd and van Oostrom (1979) found that the entire head ectoderm is initially thickened, but subsequently thins to leave the placodes in relief (they thicken additionally later in development). These morphological data suggest that placodes arise in embryogenesis from a common anlage lying between the neural plate and epidermis. This anlage could be formed by a mechanism similar to that underlying the initial induction of the neural crest (reviewed in Baker and Bronner-Fraser, 1997a; also see Neave et al., 1997). If this is the case, it could account for some of the similarities between placodal cells and neural crest cells (reviewed in Baker and Bronner-Fraser, 1997b). As more is learned about the induction of different placodes, it will be possible to test this hypothesis.

\section{Conclusion}

Pax-3 induction in the presumptive opV placode at the midbrain level begins before the 3-ss. The whole head ectoderm rostral to the first somite is competent at the 3 -ss to respond to the midbrain-level Pax-3-inducing activity, but competence is rapidly lost by the otic region. Pax-3 specification does not occur significantly before Pax-3 expression. The inducing activity is broadly distributed along the entire neuraxis and the neural tube can directly induce Pax3 expression in competent ectoderm. The caudal border of Pax3 expression in the opV placode may be defined by an inhibitor at the r2,3 level. Our detailed analysis of this induction is the first such for any of the solely neurogenic placodes.

We are very grateful to Dr Andy Groves for discussion and helpful comments on the manuscript. This work was supported by USPHS NS34671 and NS36585 (M. B. F.), and by Human Frontier Science Program Fellowship LT-63/96 and American Heart Association Fellowship 1171-FI1 (C. V. H. B.).

\section{REFERENCES}

Altmann, C. R., Chow, R. L., Lang, R. A. and Hemmati-Brivanlou, A. (1997). Lens induction by Pax-6 in Xenopus laevis. Dev. Biol. 185, 119-123. Artinger, K. B. and Bronner-Fraser, M. (1993). Delayed formation of the floor plate after ablation of the avian notochord. Neuron 11, 1147-1161.

Baker, C. V. H. and Bronner-Fraser, M. (1997a). The origins of the neural crest. Part I: Embryonic induction. Mech. Dev. 69, 3-11.

Baker, C. V. H. and Bronner-Fraser, M. (1997b). The origins of the neural crest. Part II: An evolutionary perspective. Mech. Dev. 69, 13-29. 
Baker, C. V. H., Torpey, N. B., Sharpe, C. R., Heasman, J. and Wylie, C. C. (1995). A Xenopus c-kit-related receptor tyrosine kinase expressed in migrating stem cells of the lateral line system. Mech. Dev. 50, 217-222.

Baker, C. V. H., Bronner-Fraser, M., Le Douarin, N. M. and Teillet, M.A. (1997). Early- and late-migrating cranial neural crest cell populations have equivalent developmental potential in vivo. Development 124, 30773087.

Couly, G. F. and Le Douarin, N. M. (1985). Mapping of the early neural primordium in quail-chick chimeras I. Dev. Biol. 110, 422-439.

Couly, G. and Le Douarin, N. M. (1990). Head morphogenesis in embryonic avian chimeras: evidence for a segmental pattern in the ectoderm corresponding to the neuromeres. Development 108, 543-558.

D'Amico-Martel, A. and Noden, D. M. (1983). Contributions of placodal and neural crest cells to avian cranial peripheral ganglia. Am. J. Anat. 166, 445468.

García-Martinez, V., Alvarez, I. S. and Schoenwolf, G. C. (1993). Locations of the ectodermal and nonectodermal subdivisions of the epiblast at stages 3 and 4 of avian gastrulation and neurulation. J. Exp. Zool. 267, 431-446.

Graham, A., Francis-West, P., Brickell, P. and Lumsden, A. (1994). The signalling molecule BMP4 mediates apoptosis in the rhombencephalic neural crest. Nature 372, 684-686.

Graham, A., Heyman, I. and Lumsden, A. (1993). Even-numbered rhombomeres control the apoptotic elimination of neural crest cells from odd-numbered rhombomeres in the chick hindbrain. Development 119, 233245.

Graham, A., Köntges, G. and Lumsden, A. (1996). Neural crest apoptosis and the establishment of craniofacial pattern: an honorable death. Mol. Cell. Neurosci. 8, 76-83.

Grainger, R. M. (1992). Embryonic lens induction: shedding light on vertebrate tissue determination. Trends Genet. 8, 349-355.

Grainger, R. M. (1996). New perspectives on embryonic lens induction. Semin. Cell. Dev. Biol. 7, 149-155.

Gurdon, J. B. (1987). Embryonic induction - molecular prospects. Development 99, 285-306.

Hamburger, V. (1961). Experimental analysis of the dual origin of the trigeminal ganglion in the chick embryo. J. Exp. Zool. 148, 91-117.

Hamburger, V. and Hamilton, H. L. (1951). A series of normal stages in the development of the chick embryo. J. Morphol. 88, 49-92.

Herbrand, H., Guthrie, S., Hadrys, T., Hoffmann, S., Arnold, H.-H., Rinkwitz-Brandt, S. and Bober, E. (1998). Two regulatory genes, $c N k x 5$ 1 and $c P a x 2$, show different responses to local signals during otic placode and vesicle formation in the chick embryo. Development 125, 645-654.

Jacobson, A. G. and Sater, A. K. (1988). Features of embryonic induction. Development 104, 341-359.

Knouff, R. A. (1935). The developmental pattern of ectodermal placodes in Rana pipiens. J. Comp. Neurol. 62, 17-71.

Le Douarin, N. M., Fontaine-Perus, J. and Couly, G. (1986). Cephalic ectodermal placodes and neurogenesis. Trends Neurosci. 9, 175-180.

Lee, V. M., Carden, M. J., Schlaepfer, W. W. and Trojanowski, J. Q. (1987). Monoclonal antibodies distinguish several differentially phosphorylated states of the two largest rat neurofilament subunits (NF-H and NF-M) and demonstrate their existence in the normal nervous system of adult rats. $J$. Neurosci. 7, 3474-3488.

Metscher, B. D., Northcutt, R. G., Gardiner, D. M. and Bryant, S. V. (1997). Homeobox genes in axolotl lateral line placodes and neuromasts. Dev. Genes Evol. 207, 287-295.

Miyake, T., von Herbing, I. H. and Hall, B. K. (1997). Neural ectoderm, neural crest and placodes: contribution of the otic placode to the ectodermal lining of the embryonic opercular cavity in Atlantic cod (Teleostei). $J$. Morphol. 231, 231-252.

Muske, L. E. (1993). Evolution of gonadotropin-releasing hormone (GnRH) neuronal systems. Brain Behav. Evol. 42, 215-230.

Neave, B., Holder, N. and Patient, R. (1997). A graded response to BMP-4 spatially coordinates patterning of the mesoderm and ectoderm in the zebrafish. Mech. Dev. 62, 183-195.

Sechrist, J., Nieto, M. A., Zamanian, R. T. and Bronner-Fraser, M. (1995) Regulative response of the cranial neural tube after neural fold ablation: spatiotemporal nature of neural crest regeneration and up-regulation of Slug Development 121, 4103-4115.

Slack, J. M. W. (1991). From Egg to Embryo: Regional Specification in Early Development. 2nd ed. (ed. P. W. Barlow, D. bray, P. B. Green and J. M. W. Slack). Cambridge: Cambridge University Press.

Smith, J. L. and Schoenwolf, G. C. (1987). Cell cycle and neuroepithelial cell shape during bending of the chick neural plate. Anat. Rec. 218, 196206.

Sommer, L., Ma, Q. and Anderson, D. J. (1996). neurogenins, a novel family of atonal-related bHLH transcription factors, are putative mammalian neuronal determination genes that reveal progenitor cell heterogeneity in the developing CNS and PNS. Mol. Cell. Neurosci. 8, 221-241.

Stark, M. R., Sechrist, J., Bronner-Fraser, M. and Marcelle, C. (1997). Neural tube/ectoderm interactions are required for trigeminal placode formation. Development 124, 4287-4295.

Streit, A., Sockanathan, S., Pérez, L., Rex, M., Scotting, P. J., Sharpe, P. T., Lovell-Badge, R. and Stern, C. D. (1997). Preventing the loss of competence for neural induction: HGF/SF, L5 and Sox-2. Development 124, 1191-1202.

van Wijhe, J. W. (1883). Uber die Mesodermsegmente und die Entwicklung der Nerven des Selachierkopfes. Verh. Acad. Setensch. (Amsterdam) 22(E), $1-50$.

Verwoerd, C. D. A. and van Oostrom, C. G. (1979). Cephalic neural crest and placodes. Adv. Anat. Embryol. Cell Biol. 58, 1-75.

Vogel, K. S. and Davies, A. M. (1993). Heterotopic transplantation of presumptive placodal ectoderm changes the fate of sensory neuron precursors. Development 119, 263-276.

Walther, C. and Gruss, P. (1991). Pax-6, a murine paired box gene, is expressed in the developing CNS. Development 113, 1435-1449.

Webb, J. F. and Noden, D. M. (1993). Ectodermal placodes: contributions to the development of the vertebrate head. Amer. Zool. 33, 434-447.

Zygar, C. A., Cook, T. L. J. and Grainger, R. M. (1998). Gene activation during early stages of lens induction in Xenopus laevis. Development $\mathbf{1 2 5}$, 3509-3519. 